\title{
Mitochondria Targeted Protein-Ruthenium Photosensitizer for Efficient Photodynamic Applications
}

\author{
Sabyasachi Chakrabortty, ${ }^{\dagger}, \|$ Bikram Keshari Agrawalla, ${ }^{\dagger, \|}$ Anne Stumper, ${ }^{\ddagger}$ Naidu M Vegi, ${ }^{\S}$
} Stephan Fischer, ${ }^{\dagger}$ Christian Reichardt, ${ }^{\#}$ Michael Kögler, ${ }^{\dagger}$ Benjamin Dietzek, ${ }^{\#}$ Michaela Feuring-Buske, ${ }^{\perp, \S}$ Christian Buske, ${ }^{\S}$ Sven Rau, ${ }^{*, \neq}$ and Tanja Weil ${ }^{*},+, \|_{\odot}$

${ }^{\dagger}$ Department of Organic Chemistry III, ${ }^{\ddagger}$ Institute of Inorganic Chemistry I, and ${ }^{\S}$ Institute for Experimental Cancer Research, Comprehensive Cancer Center, Ulm University, Albert-Einstein-Allee 11, $89081 \mathrm{Ulm}$, Germany

"Max-Planck-Institute for Polymer Research, Ackermannweg 10, 55128 Mainz, Germany

${ }^{\perp}$ Department of Internal Medicine III, University Hospital Ulm, Albert-Einstein Allee 23, 89081, Ulm, Germany

\#Department of Functional Interfaces, Leibniz Institute of Photonic Technology (IPHT) Jena, Albert-Einstein-Straße 9, 07745 Jena, Germany

\section{Supporting Information}

ABSTRACT: Organelle-targeted photosensitization represents a promising approach in photodynamic therapy where the design of the active photosensitizer (PS) is very crucial. In this work, we developed a macromolecular PS with multiple copies of mitochondria-targeting groups and ruthenium complexes that displays highest phototoxicity toward several cancerous cell lines. In particular, enhanced anticancer activity was demonstrated in acute myeloid leukemia cell lines, where significant impairment of proliferation and clonogenicity occurs. Finally, attractive two-photon absorbing properties further underlined the great significance of this PS for mitochondria targeted PDT applications in deep tissue cancer therapy.

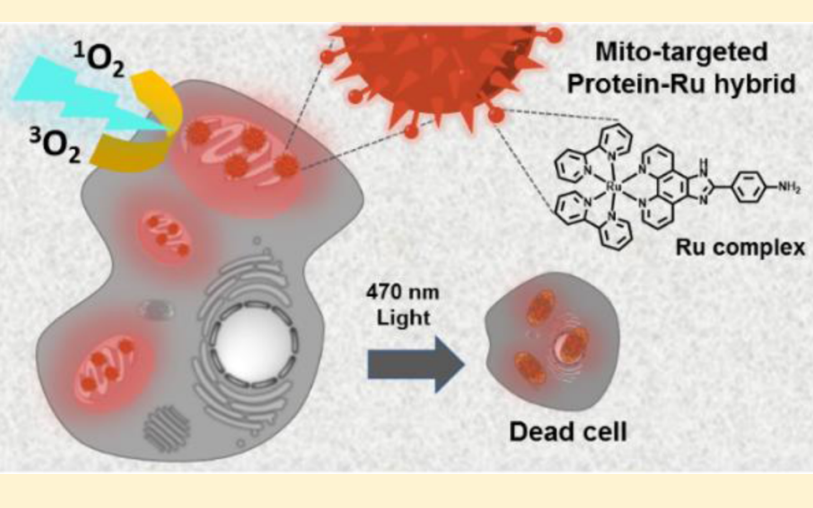

photophysical and photochemical features as well as their DNA intercalation capacity and protein binding motifs. ${ }^{3,8,9}$ In particular, organo-ruthenium complexes coordinated by polypyridyl ligands exhibited promising anticancer activity when irradiated with light. ${ }^{10}$ Their high population of the triplet metal-to-ligand charge-transfer state $\left({ }^{3} \mathrm{MLCT}\right)$, due to the heavy atom effect, produces large ${ }^{1} \mathrm{O}_{2}$ yields, while the solubility of these complexes can be modified by adjusting the counterions. For example, the $\left[\mathrm{Ru}(\mathrm{bpy})_{3}\right]^{2+}$ derivative TLD1433 recently entered phase I clinical trials as the first $\mathrm{Ru}$-based PS, due to its potential in effectively producing ${ }^{1} \mathrm{O}_{2}{ }^{11}$ In combination with a targeting peptide providing high binding affinity for membrane proteins, a Ru-PS has been achieved with high selectivity for certain cancer cells. ${ }^{12}$ However, in order to further advance PDT for therapy, several limitations of the PS still need to be solved such as their low cellular uptake efficiency, low extinction coefficients, and only moderate cellular toxicity. Herein, we present a macromolecular approach to improve phototoxicity and efficacy of the PS by synergistic combination of $\mathrm{Ru}$-complexes on a protein carrier scaffold decorated with subcellular mitochondria targeting groups.

Received: December 31, 2016

Published: January 18, 2017 
Scheme $1^{a}$

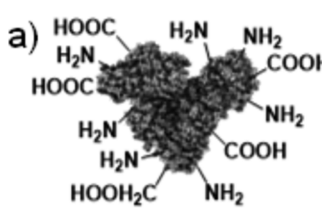

HSA

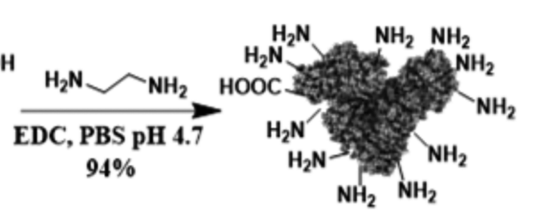

cHSA

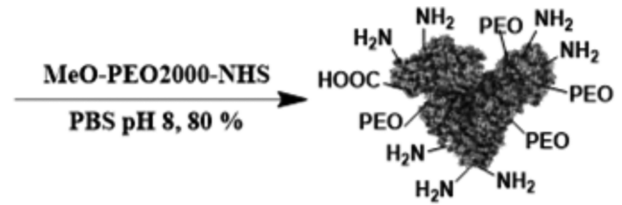

cHSA-PEO
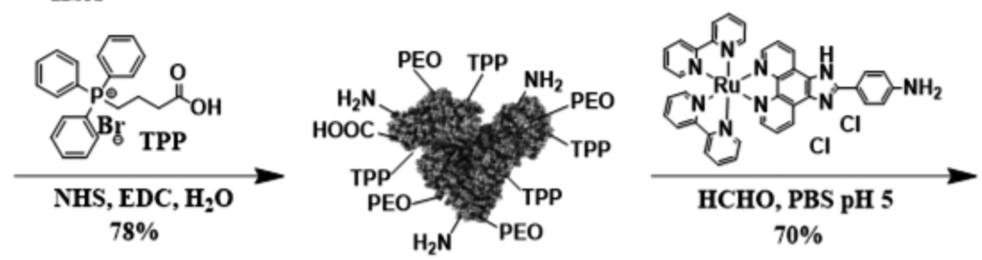

CHSA-PEO-TPP
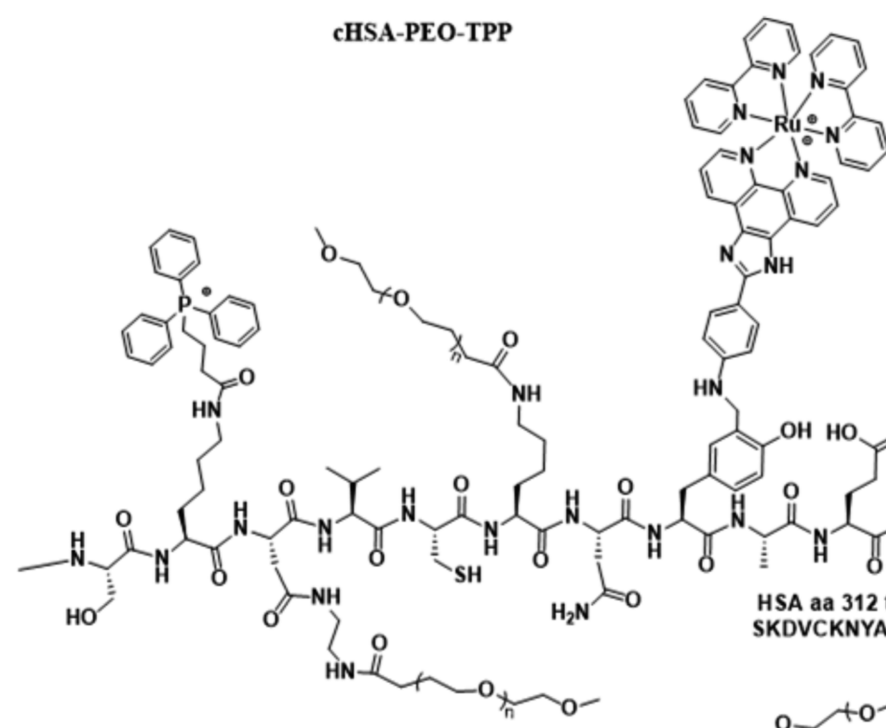

b)

a (a) Synthetic scheme of the cHSA-PEO-TPP-Ru transporter based on HSA. Subsequent functionalities were conjugated at different reactive sites of the HSA backbone. (b) Schematic illustration of a part of the HSA polypeptide sequence exemplary with the PEO, TPP groups attached to, e.g., lysine and $\mathrm{Ru}$ conjugated to tyrosine residues.

Mitochondria, as indispensable organelles responsible for cell respiration, emerge as promising pharmacological target in clinical applications for the detection, inhibition and treatment of various diseases such as cancer or neurodegenerative diseases, due to their crucial role in mediating cell apoptosis. ${ }^{13,14}$ Until now, only little is known of the balance of reactive oxygen species in cancer cells and their survival mechanisms that effect mitochondria function. There have been many attempts to target cancer cells via signaling pathways. ${ }^{15}$ However, drug strategies targeting the mitochondrial metabolism are scarce and though present, treatment approaches were not achieved at low drug concentrations. The conjugation of PS with mitochondria targeting groups is considered an emerging strategy to enhance cellular toxicity by localizing the PS at the

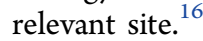

Herein, we investigate efficient growth inhibition in an acute myeloid leukemia (AML) cell line by a macromolecular PS targeting mitochondria, which are known as "power house of the cell" and that are central organelles for tumor growth. ${ }^{17}$ AML is an aggressive disease which still leads to death in up to 8 of 10 patients outside of clinical trials. It is characterized by aberrant high proliferation and increase in immature blasts and progenitors due to blockade in cell differentiation. Leukemic cells initially respond to chemotherapy. However, relapse is common and in most cases fatal. Thus, there is an urgent need to develop innovative therapeutic concepts, which target leukemic cells, but spare normal hematopoietic stem and progenitor cells.

We propose a bioinspired strategy that converts the blood plasma protein serum albumin (HSA) into an efficient nanotransporter for phototoxic drug molecules, ${ }^{18-21}$ providing synergistic features due to the molecular design. The resultant nanotransporter denoted cHSA-PEO-TPP-Ru exhibited significantly improved photophysical properties and enhanced ${ }^{1} \mathrm{O}_{2}$ quantum yields as compared to the bare $\mathrm{Ru}$ complex as well as excellent mitochondria-specific colocalization. Efficient phototoxicity of cHSA-PEO-TPP-Ru already at nanomolar concentrations were achieved, which was attributed to synergistic effects from the high number of $\mathrm{Ru}$-complexes as well as organelle-targeting features of the biopolymer. To the best of our knowledge, cHSA-PEO-TPP-Ru reported herein displays the lowest $\mathrm{IC}_{50}$ value for cancerous cell lines and therefore highest cytotoxicity of a Ru-containing molecule reported to date in cellular studies. Efficient inhibition of growth in an AML cell line was observed, with preferential killing of leukemic cells compared to normal bone marrow cells, suggesting a therapeutic window for this compound in AML. Furthermore, Two-photon absorption features of cHSA-PEO-TPP-Ru under- 
a)

\section{然}
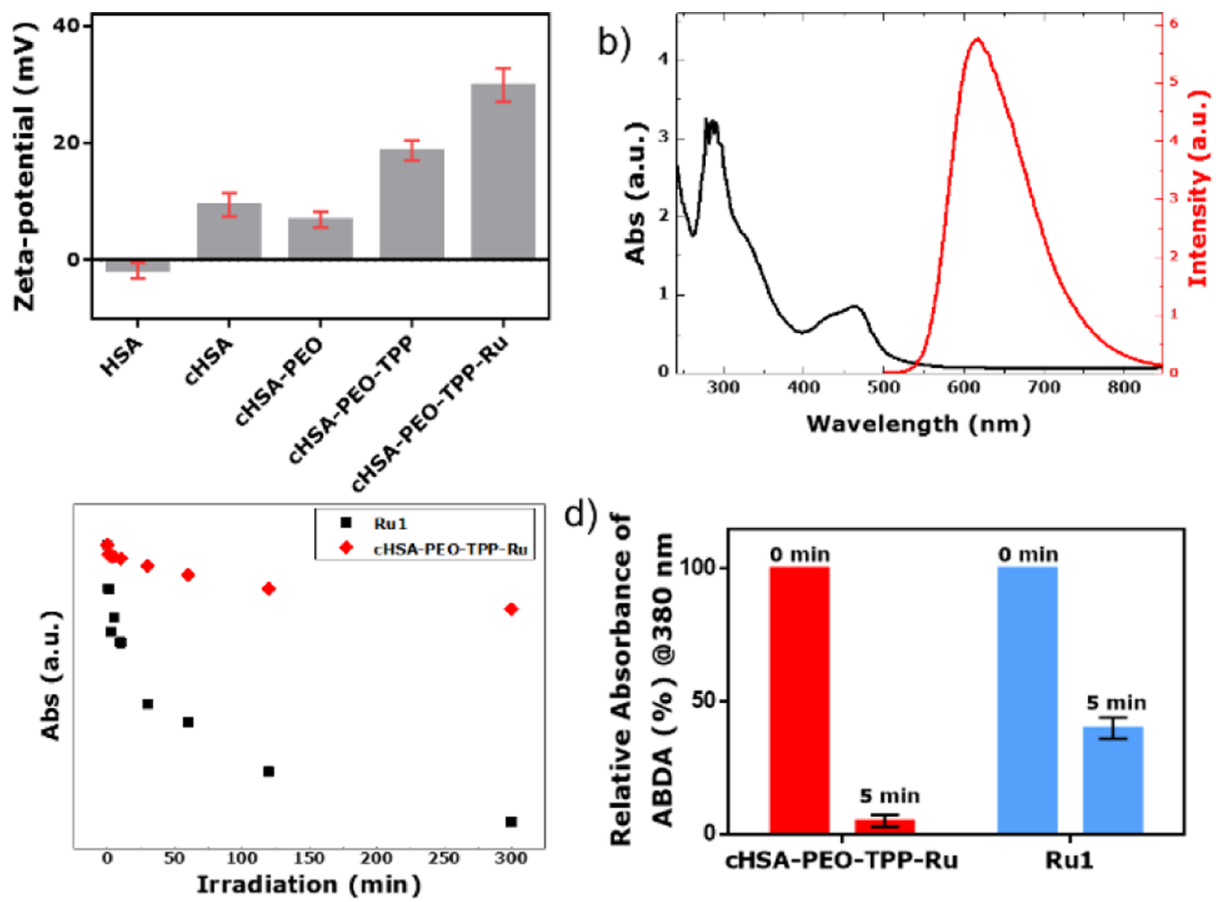

c)

e)

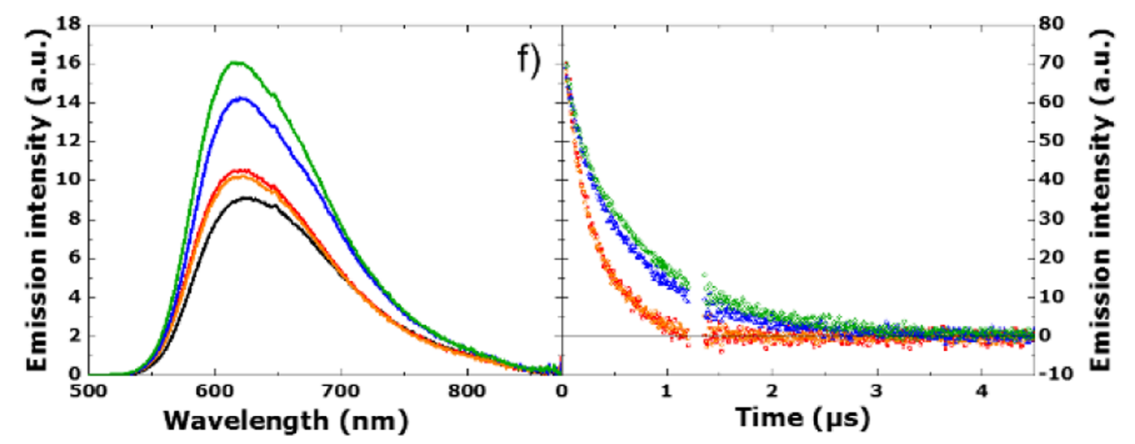

Figure 1. (a) Zeta potential of different bioconjugates. (b) Typical absorbance and emission spectra of cHSA-PEO-TPP-Ru, where characteristic peaks of the Ru complexes are preserved in cHSA-PEO-TPP-Ru. (c) Comparison of the photostability of the Rul and cHSA-PEO-TPP-Ru based on the absorbance decay under continuous irradiation over extended time periods. (d) ${ }^{1} \mathrm{O}_{2}$ production yield of cHSA-PEO-TPP-Ru and bare Ru complex (Ru1), as obtained from the photobleaching of the characteristic absorption peak @380 nm of ABDA (100 $\mu$ M) during irradiation with 470 $\mathrm{nm}$ LED light $\left(\sim 20 \mathrm{~mW} / \mathrm{cm}^{2}, 5 \mathrm{~min}\right)$ in PBS (1×, pH 7.4) based on the same optical density in their first absorption peak. (e) Steady state emission spectra $\left(\lambda_{\mathrm{ex}}=460 \mathrm{~nm}\right)$ of Rul complex in water (red), Rul complex in simulated body fluid (orange), cHSA-PEO-TPP-Ru in water (blue), cHSAPEO-TPP-Ru in simulated body fluid (green), and $\mathrm{Ru}(\mathrm{bpy})_{3}$ (black) as reference at same optical density. (f) Emission lifetime experiments upon excitation at $460 \mathrm{~nm}$. For the compounds, the same color code is used and an artifact region between 1.2 and $1.35 \mu$ s has been removed.

line its great potential as two-photon activated photosensitizer for in vivo PDT.

\section{RESULTS AND DISCUSSION}

Synthesis and Characterization of cHSA-PEO-TPP-Ru. The synthesis of the cHSA-PEO-TPP-Ru is depicted in Scheme 1. HSA serves as biocompatible and biodegradable backbone providing many reactive carboxylic acid, amino and hydroxyl groups originating from the respective amino acid side chains of HSA that can be further chemically functionalized. First, all accessible carboxylic acid groups were transformed into primary amino groups by applying ethylenediamine and 1-ethyl-3-(3(dimethylamino)propyl) carbodiimide (EDC) to increase the total number of reactive amino groups for further conjugations and enhance interactions of the biopolymer with negatively charged cellular membranes as published previously. ${ }^{22}$ After purification through dialysis, globular, polycationic cHSA was obtained, which facilitates cellular uptake by Clathrin-mediated endocytosis. $^{23}$ To impart water solubility and reduce nonspecific interactions, poly(ethylene oxide) (PEO-2000) side chains were introduced by reacting $\alpha$-methoxy- $\omega$-carboxylic acid succinimidyl ester polyethylene(oxide) (NHS-PEO) with cHSA and subsequent washing five times by ultrafiltration with vivaspin 20 (MWCO 30K) centrifuge tubes. According to Maldi-ToF, about 20 PEO chains were attached to cHSA. ${ }^{24}$ To achieve mitochondria targeting, multiple units of TPP were reacted to the free amino groups of cHSA-PEO. Briefly, we mixed EDC-NHS activated (3-carboxypropyl) triphenylphosphonium bromide (TPP) ester with cHSA-PEO to yield cHSAPEO-TPP hybrid with approximately 34 TPP units attached. Finally, the resulting product was washed five times through vivaspin 20 (MWCO $30 \mathrm{~K}$ ) centrifugal concentrator for further usage.

Mannich reactions have been reported to modify phenol groups of tyrosine side chains of proteins with high selectivity. ${ }^{25}$ Therefore, the aniline-modified Ru-complex denoted "Ru1" was 
selected as it allows bioconjugation of these sterically demanding Ru-complexes following a Mannich-type reaction in aqueous solution. Compared to many known $\mathrm{Ru}$-complexes, $\mathrm{Ru} 1$ reveals exceptional water solubility of $153 \mathrm{mg} / \mathrm{mL}$ making it well-suitable for protein modifications under mild conditions. HSA provides 18 tyrosine groups and half are located exposed to the surface according to computer simulations. A threecomponent Mannich-type coupling reaction was carried out applying a mixture of cHSA-PEO-TPP, formaldehyde ( $\mathrm{HCHO})$ and the Ru-complex (Ru1, SI) yielding the desired cHSA-PEO-TPP-Ru as yellowish-brown solution in $70 \%$ isolated yield. Excessive amounts of the reactants (e.g., unreacted $\mathrm{Ru}$ complex and $\mathrm{HCHO}$ ) were removed by ultrafiltration with vivaspin 20 (MWCO 30K) centrifugation tubes until no free Ru-complex was detected in the elution media anymore. A control experiment was performed without formaldehyde and no $\mathrm{Ru}$ attachment was observed (Figure SI1 ), indicating low tendency of Ru-complexes for unspecific adsorption into the hydrophobic pockets of HSA.

MALDI-ToF characterization of the chemically modified globular proteins was accomplished for each reaction step. The respective MALDI-ToF mass spectra are depicted in Figure SI2, indicating that about $10 \mathrm{Ru}$-complexes were loaded to cHSAPEO-TPP. The resulting cHSA-PEO-TPP-Ru shows solubility of $>65 \mathrm{mg} / \mathrm{mL}$ as well as high stability at $4{ }^{\circ} \mathrm{C}$ for more than eight months (long-term storage studies are still ongoing). In order to evaluate the hydrodynamic radius of cHSA-PEO-TPP$\mathrm{Ru}$ in solution and cell culture medium, DLS studies were accomplished. An average hydrodynamic radius of about $40 \mathrm{~nm}$ was obtained (Figure SI-3). Polymer sizes in this range are considered favorable for accumulation in tumor tissues via the EPR effect, ${ }^{26}$ although this effect is currently under debate. ${ }^{27}$ Zeta-potential of cHSA-PEO-TPP-Ru (Figure 1a) reveals a positive surface charge facilitating interactions with negatively charged cellular membranes to induce endocytosis.

Photostability and ${ }^{1} \mathrm{O}_{2}$ Yield of cHSA-PEO-TPP-Ru. cHSA-PEO-TPP-Ru exhibited characteristic absorption and emission maxima at around 460 and $617 \mathrm{~nm}$, respectively (Figure $1 b$ ), similar to the starting complex Ru1, cHSA-PEOTPP-Ru revealed no alteration of the metal-to-ligand charge transfer band (MLCT) during the reaction. Furthermore, the emission spectrum of cHSA-PEO-TPP-Ru was insensitive to the composition of the solvent, i.e., the emissive excited state did not respond to environmental changes such as variations of the solvent composition. To evaluate the photostability of cHSA-PEO-TPP-Ru, photobleaching experiments were conducted in water.

Previous publications have reported that the photoinstability of $\mathrm{Ru}(\mathrm{II})$ polypyridyl complexes including $\left[\mathrm{Ru}(\mathrm{bpy})_{3}\right]^{2+}$ limits their PDT applications ${ }^{28-30}$ and functionalized nanoparticles revealed higher photostability compared to the amine containing $\mathrm{Ru}(\mathrm{II})$ polypyridyl complexes. ${ }^{31}$ Figure 1c shows the greatly improved photostability of cHSA-PEO-TPP-Ru compared to the Rul, which was recorded under the same conditions. Even over greatly extended irradiation times $(65 \mathrm{~h})$, cHSA-PEO-TPP-Ru remained remarkably stable (with only $36 \%$ decrease) in comparison to Rul. For the latter, significantly decreased absorbance of about $76 \%$ was detected after $18 \mathrm{~h}$ already (Figure SI-4). The luminescence of both compounds was observed over time and loss of emission intensity of about $68 \%$ and $89 \%$ occurred after $300 \mathrm{~min}$ for cHSA-PEO-TPP-Ru and Ru1, respectively. Obviously, photo- chemical stability of the Ru-complex in the biopolymer has significantly increased compared to Rul.

PDT relies on efficient production of singlet oxygen in cellular environments. In order to monitor the generation of ${ }^{1} \mathrm{O}_{2}$ in a quantitative fashion, we performed ${ }^{1} \mathrm{O}_{2}$ production efficiency tests at four different LED sources, e.g., $770 \mathrm{~nm}, 625$ $\mathrm{nm}, 525 \mathrm{~nm}$ and $470 \mathrm{~nm}$ as reported by us previously. ${ }^{12}$ The singlet oxygen sensor 9,10-anthracenediyl-bi(methylene)dimalonic acid (ABDA) was applied, which forms an endoperoxide of $A B D A$ in the presence of ${ }^{1} \mathrm{O}_{2}$, thus decreasing ABDA absorption and providing a valuable means of direct monitoring ${ }^{1} \mathrm{O}_{2}$ production (Figure SI-5). According to these measurements, $470 \mathrm{~nm}$ proved to be most efficient excitation source (Figure SI-6). cHSA-PEO-TPP-Ru and cHSA-PEO-TPP as control were mixed separately with $100 \mu \mathrm{M}$ of ABDA in PBS buffer and then irradiated with a $470 \mathrm{~nm}$ LED array $(P=20 \pm$ $2 \mathrm{~mW} / \mathrm{cm}^{2}$ ) for $5 \mathrm{~min}$. As described in Figure SI-9a, cHSAPEO-TPP-Ru produced ${ }^{1} \mathrm{O}_{2}$ very efficiently, whereas cHSAPEO-TPP remained inactive.

Equimolar concentrations of $\mathrm{Ru}$ in cHSA-PEO-TPP-Ru and $\mathrm{Rul}$ were used for all further experiments to compare the photophysical and biological features of $\mathrm{Ru}$ in the cHSA-PEOTPP-Ru bioconjugate versus Rul. As depicted in Figure 1d, the ${ }^{1} \mathrm{O}_{2}$ production for Rul and cHSA-PEO-TPP-Ru was measured at the same optical density at $460 \mathrm{~nm}$, e.g., at similar $\mathrm{Ru}$ concentrations. About $\sim 8$-fold improved reduction of the ABDA absorption peak @380 nm has been achieved for a single $\mathrm{Ru}$ molecule attached to the biopolymer compared to a single Rul complex (details included in the Supporting Information). Again, molar concentrations have been used for comparison. We believe that the synergistic interaction between closely spaced multiple Rul in the lipophilic protein backbone might be the reason for this finding.

Photophysical Properties of cHSA-PEO-TPP-Ru. The photophysical properties of the model Rul-complex and cHSAPEO-TPP-Ru were investigated by steady state emission spectroscopy following a literature reported procedure. ${ }^{32,33}$ The photochemistry of Ru-complexes is highly sensitive to their respective environments. Emission spectra of both compounds were identical in water and simulated body fluid solutions (Figure 1e). A slightly higher emission quantum yield of cHSAPEO-TPP-Ru (using Ru(bpy) ${ }_{3}$ as standard) was observed upon increasing the ionic strength of the solution, i.e., by comparing the simulated body fluid $\left(\Phi_{\text {cHSA-PEO-TPP-Ru, body fluid }}=6.7 \%\right)$ to water $\left(\Phi_{\text {cHSA-PEO-TPP-Ru, water }}=6.1 \%\right)$. On the contrary, this trend was not observed for the Rul $\left(\Phi_{\mathrm{Ru} \text {, water }}=4.8 / \Phi_{\mathrm{Ru} \text {, body fluid }}\right.$ $=4.5 \%)$ ). The generally higher fluoresecence quantum yields of cHSA-PEO-TPP-Ru and $\mathrm{Ru} 1$ indicate that nonradiative deactivation paths were suppressed in the cHSA-PEO-TPP$\mathrm{Ru}$ biopolymer. This might be attributed to a sterically hindered rotation of the imidazole-phenyl bond due to conjugation to the bulky HSA protein. In line with this assignment of blocking nonradiative decay paths, we observed the prolongation of the triplet excited state lifetime in cHSA-PEO-TPP-Ru compared to Rul (Figure 1f). Assuming a monoexponential decay to analyze the respective luminescence kinetics, the lifetime was increased from $300 \mathrm{~ns}$ for both solutions of $\mathrm{Rul}$ to $625 / 735 \mathrm{~ns}$ for cHSA-PEO-TPP-Ru in water/simulated body fluid.

Intracellular Optical Microscopy Imaging. We examined the intracellular localization of cHSA-PEO-TPP-Ru into a human cervical cancer cell line, HeLa cells, as model system, by laser scanning confocal microscopy. Typically, these cells were incubated with low concentrations of cHSA-PEO-TPP-Ru (500 
$\mathrm{nM}$ ) for about $4 \mathrm{~h}$ before images were recorded. To observe MLCT absorbance from the metal complex inside living cells, laser excitation at $458 \mathrm{~nm}$ was applied. The emission window was adjusted in the range of $530-710 \mathrm{~nm}$. After staining with various subcellular organelle dyes (Figure 2) with selectivity for

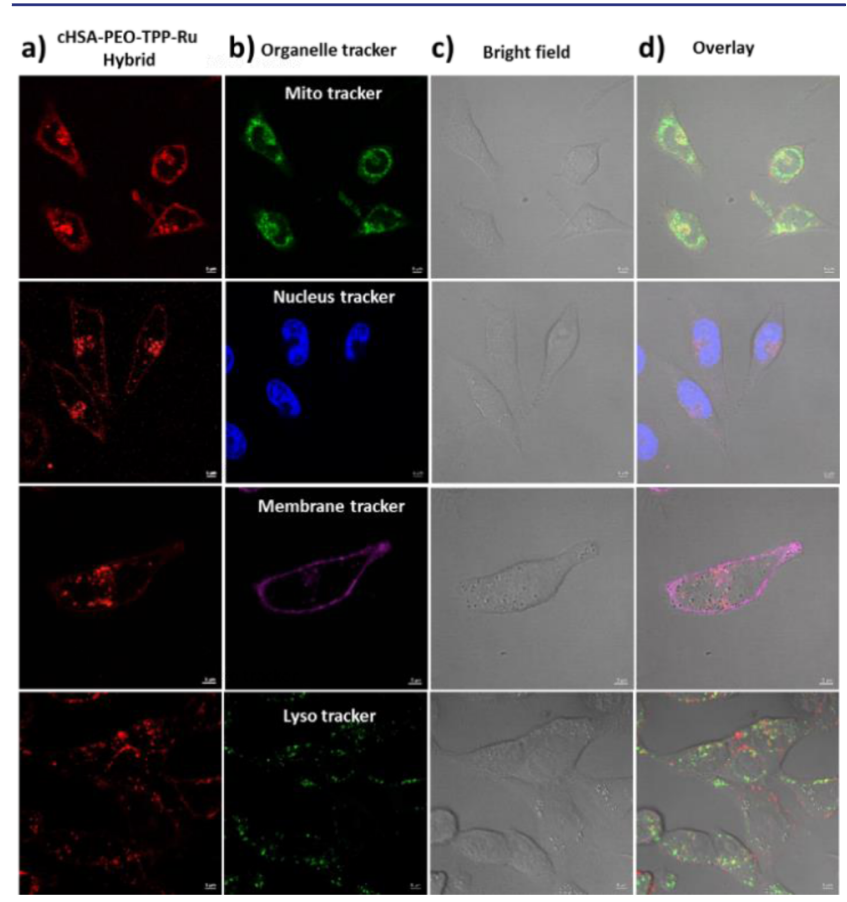

Figure 2. Confocal microscopy images of HeLa cells incubated with cHSA-PEO-TPP-Ru and treated with commercial organelle trackers. Overlay images and colocalization analysis of cells stained with mitochondria (0.88), nucleus (0.07), membrane (0.2), and lysosome (0.3) markers indicated that cHSA-PEO-TPP-Ru localized in mitochondria. (a) cHSA-PEO-TPP-Ru emission, (b) emission from the organelle trackers, (c) corresponding bright field images, and (d) overlay of all three images.

membranes (Cell Mask Deep Red Plasma Membrane Stain), the nucleus (Hoechst 33342 Solution), mitochondria (Mito Lite Blue FX490, Figure SI-7c), and lysosomes (Lyso Tracker Green DND-26), we found a clear colocalization of cHSAPEO-TPP-Ru in mitochondria (Pearson's coefficient 0.88), where they were mostly situated in the cytosol outside the nuclear region (Pearson's coefficient 0.07). cHSA-PEO-TPP$\mathrm{Ru}$ was transported rapidly across the membrane and accumulated in the cytosol and no localization in membranes (Pearson's coefficient 0.2) or in lysosomes (Pearson's coefficient 0.3 ) was observed.

Light induced cellular toxicity evaluation. To evaluate the cellular uptake efficiency of cHSA-PEO-TPP-Ru in vitro, we incubated HeLa cells with the biopolymer over different time intervals ranging from 1-240 min. Flow cytometry measurements revealed that the fluorescence intensity of treated HeLa cells reached a saturation level after $200 \mathrm{~min}$ of biopolymer incubation (Figure SI-7). Thus, $240 \mathrm{~min}$ was selected as the appropriate incubation time to ascertain maximum cellular uptake. To identify the optimum concentration of cHSA-PEOTPP-Ru, HeLa cells were incubated with $0-2 \mu \mathrm{M}$ cHSA-PEOTPP-Ru for $240 \mathrm{~min}$ after $5 \mathrm{~min}$ irradiation with $470 \mathrm{~nm}$ LED light $\left(\sim 20 \mathrm{~mW} / \mathrm{cm}^{2}\right)$. Here, the applied light dose was comparable to the reported dosage of established photosensitizing drugs reported. ${ }^{34}$ Also, we used commercially available TOX-8 dye (Sigma-Aldrich), to obtain the number of viable cells quantitatively by means of spectrophotometric measurement in all cases of cell viability experiments, according to the manufacturer's instructions. ${ }^{35}$ A concentration dependent cytotoxicity was observed in the photo irradiated sample, whereas cells incubated with of cHSA-PEO-TPP-Ru in the dark revealed almost no cellular toxicity (Figure $3 \mathrm{a}, \mathrm{b}$ ) over the entire

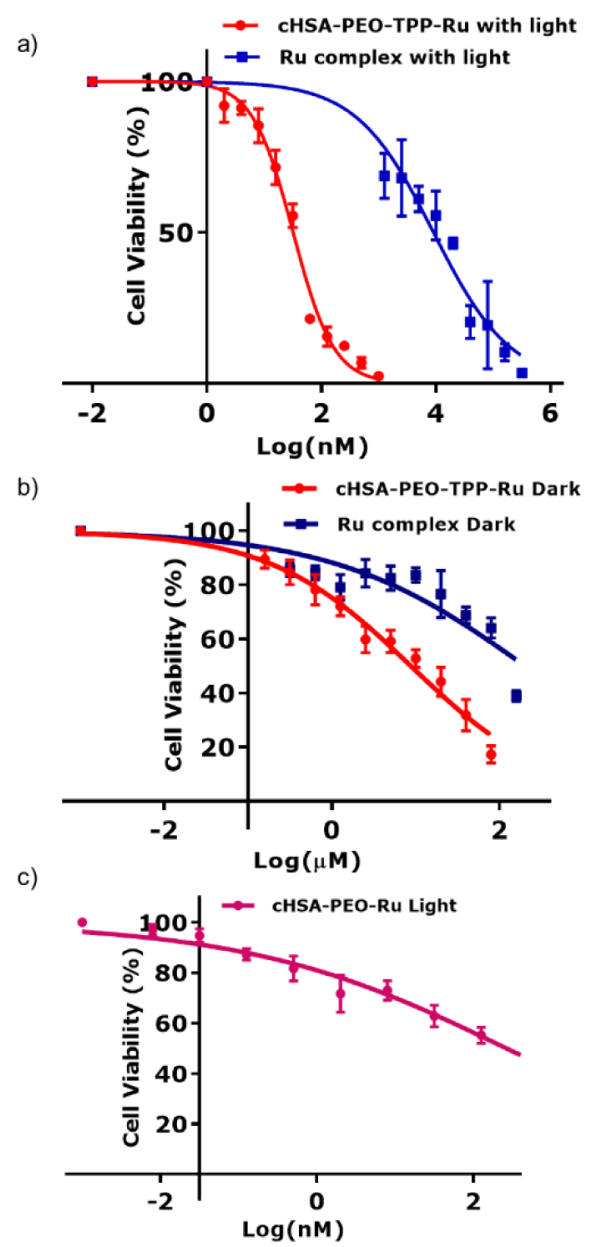

Figure 3. $(a, b)$ Logarithmic fitting curve for cell viability of cHSAPEO-TPP-Ru and bare Ru complex, over a broad concentration range with and without light. (c) Logarithmic fitting curve for cell viability of cHSA-PEO-Ru complex with light, where mitochondria targeting TPP group were absent. For all of the above experiments, HeLa cells were exposed to a $470 \mathrm{~nm}$ LED lamp $\left(\sim 20 \mathrm{~mW} / \mathrm{cm}^{2}\right)$ for $5 \mathrm{~min}$ for light irradiation. cHSA-PEO-TPP-Ru reveals low dark toxicity $\left(\mathrm{IC}_{50}=9 \pm 2\right.$ $\mu \mathrm{M})$ but very high phototoxicity $\left(\mathrm{IC}_{50}=34.9 \pm 2 \mathrm{nM}\right)$ compared to $\mathrm{Ru} 1$ (dark $\mathrm{IC}_{50}=203 \pm 3 \mu \mathrm{M}$; photoirradiated $\left.\mathrm{IC}_{50}=7.7 \pm 1.3 \mu \mathrm{M}\right)$. In the absence of a TPP group, the phototoxic effect of the drug was reduced by $\sim 8$ times $\left(\mathrm{IC}_{50}=265 \pm 1.2 \mathrm{nM}\right)$.

concentration range. A very low $\mathrm{IC}_{50}$ value of $34.9 \pm 2 \mathrm{nM}$ was obtained for cHSA-PEO-TPP-Ru under light irradiation. In comparison, $\mathrm{Rul}$ had an $\mathrm{IC}_{50}$ value of only $7.7 \pm 1.3 \mu \mathrm{M}$ (Figure SI-8) indicating greatly enhanced cellular toxicity of CHSA-PEO-TPP-Ru by about 220 -fold with minimal dark toxicity. Thus, attaching multiple $\mathrm{Ru} 1$ to one protein nanocarrier yielded a nanocarrier with surprisingly high cytotoxicity with an $\mathrm{IC}_{50}$ well below the value one would expect considering an just additive effect. Considering that 10 $\mathrm{Ru}$ chromophores were attached to cHSA-PEO-TPP-Ru, a calculated $\mathrm{IC}_{50}$ value of $0.349 \mu \mathrm{M}$ could be estimated for each 
$\mathrm{Ru}$-chromophore, which is considerably lower compared to $\mathrm{Ru} 1\left(\mathrm{IC}_{50}\right.$ value $\left.7.7 \mu \mathrm{M}\right)$ alone. Next, we studied the impact of mitochondria targeting TPP groups on carrier toxicity. Analogous phototlooxicity experiments were accomplished with cHSA-PEO-Ru without TPP groups and about 8-fold lower drug toxicity was obtained (Figure $3 \mathrm{c}$ ).

The phototoxic index (PI) of all compounds was calculated, which denotes the ratio of the dark and light-exposed $\mathrm{IC}_{50}$ values. The protein hybrid cHSA-PEO-TPP without Rul was light inactive (Figure SI-9a), whereas Rul only revealed a PI of 27. The PI of cHSA-PEO-Ru without TPP groups increased to 75 and cHSA-PEO-TPP-Ru had a significantly higher PI of 250. We believe this higher value was based on our molecular design, where the multiple molecular components contribute synergistically to the observed elevated cellular toxicity.

Furthermore, we examined the phototoxicity of cHSA-PEOTPP-Ru toward various other cancerous cell lines such as CHO, MCF7 and A549. All tumor cells tested were proficiently damaged with low $\mathrm{IC}_{50}$ values in the nanomolar range, for instance, $135.2 \pm 1 \mathrm{nM}$ for CHO, $114.3 \pm 1 \mathrm{nM}$ for MCF7, and $119.1 \pm 1 \mathrm{nM}$ for the A549 cell line (Figures SI-10-12). As additional features, the HSA polypeptide backbone is fully biodegradable even after chemical modification, which should allow efficient elimination and reduced accumulation.

Colony Forming Cell (CFC) and Proliferation Assays. In order to test the efficacy of cHSA-PEO-TPP-Ru in a relevant primary cell assay, we treated an acute myeloid leukemia (AML) cell line, OCI-AML3, which reflects the biology of primary NPM1 mut - AML, comprising around 35\% of all human AML cases ${ }^{36}$ and $60 \%$ of AML with normal karyotype. ${ }^{37}$ Irradiation of 2 and 5 min induced a significant $(p<0.005)$ reduction of $44 \%$ and $84.4 \%$ of colony growth, respectively, in comparison to the treated but nonirradiated control arm (dark) as assessed by colony number in the colony forming cell (CFC) assay $(p<0.005)$. In addition to the reduction in colony number, remaining colonies were smaller in size after exposure to cHSA-PEO-TPP-Ru (Figure 4b). Furthermore, we tested cHSA-PEO-TPP-Ru for its effect on proliferation with two different concentrations (75 and 37.5 $\mathrm{nM}$ ). A significant decrease of the proliferative capacity was observed over a period of up to $72 \mathrm{~h}$ after exposure to light for 2 and $5 \mathrm{~min}$, respectively, compared to the dark control (Figure $4 c, d)$.

To determine the differential effect of cHSA-PEO-TPP-Ru on normal versus leukemic primary bone marrow (BM) cells, we performed CFC assays on healthy murine BM cells as well as on a murine AML cell line derived from BM of a mouse transplanted with a truncated version of the leukemia-specific AML1-ETO fusion gene (AML1-ETO 9a (AE9a)). ${ }^{38}$ We could document a decrease in the colony forming capacity of the leukemic AE9a cell line by $37 \%$ and $88 \%$ reduction after 2 and $5 \mathrm{~min}$ exposure to light, respectively, compared to the nonirradiated control cells (Figure SI-13). In contrast, there was only a $10 \%$ and $28 \%$ reduction of colony growth, respectively, when normal $\mathrm{BM}$ cells were treated accordingly (Figure SI-14), indicating that cHSA-PEO-TPP-Ru significantly inhibits the proliferative and clonogenic potential of primary murine AML while sparing normal bone marrow stem and progenitor cells. Previously, Sieber and co-workers have successfully demonstrated photodynamic treatment of bone marrow/leukemic cell (L1210) mixtures with visible light of 410-500 nm. ${ }^{39}$ Even though significant reduction of the leukemic cell number was shown in vitro, subsequent in vivo a)

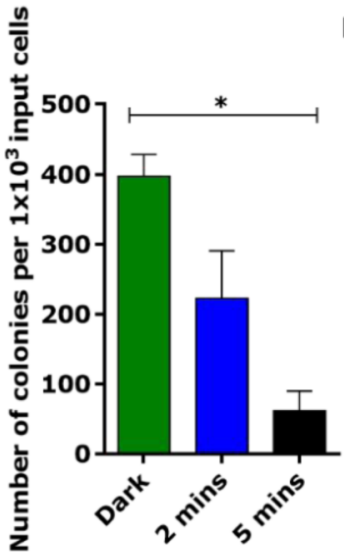

c)

d)
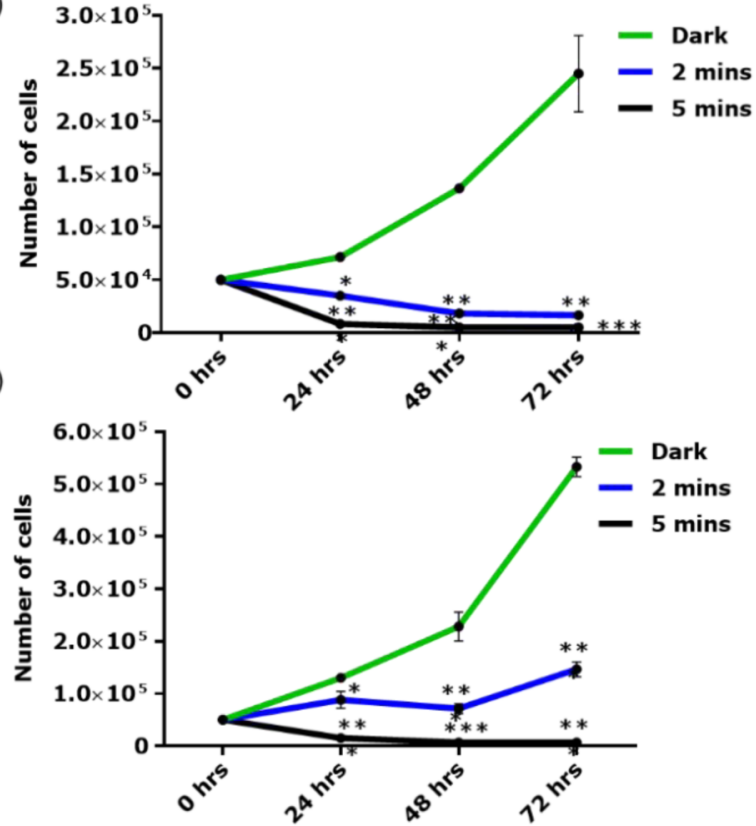

Figure 4. (a) Colony forming cell (CFC) assay of the OCI-AML3 (OA3) AML cell line ( $n=2$ in duplicates) showed reduction of colonies after 2 and 5 min exposure to light $(470 \mathrm{~nm})$ compared to the treated and nonexposed cells (dark). Bars indicate mean \pm SEM. Significance calculated by Mann-Whitney test $(*<0.05)$. (b) Morphology of the colonies in the control arm and 5 min exposure arm (4X magnification). (c,d) Proliferation assay of the OA3 cell line $(n=3)$ incubated with two different concentrations of cHSA-PEOTPP-Ru $(75 \mathrm{nM})$ and $(37.5 \mathrm{nM})$ and exposed to light for 2 and $5 \mathrm{~min}$ or treated but not exposed to light. Significance calculated by two-way ANOVA multiple test comparisons test $(*<0.05 ; * * *<0.0001)$.

studies in a mouse transplant were less successful. It was speculated that photoactive protoporphyrin IX (PpIX) had to be biosynthesized within the leukemic cells and leukemic cells within the resting phase did not perform this biosynthesis and therefore escaped this treatment. In our approach, cHSA-PEOTPP-Ru conjugate exhibits very similar PDT effects, but due to the biopolymer design no biosynthesis of the photosensitizer is required for bioactivity.

Two-Photon Active Probe. For in vivo PDT, deep-tissue penetration would be relevant to reach also tumor cells located in deeper tissue. TP microscopy has evolved as an efficient tissue imaging and therapeutic platform due to its longer wavelength excitation laser light, which offers deep tissue penetration, reduced photodamage and $3 \mathrm{D}$ and high contrast 
imaging. ${ }^{10}$ We have measured the two-photon (TP) properties of cHSA-PEO-TPP-Ru to assess its suitability as TP probe. cHSA-PEO-TPP-Ru revealed almost five times higher TP action cross section compared to the Ru complex (Figure 5a).

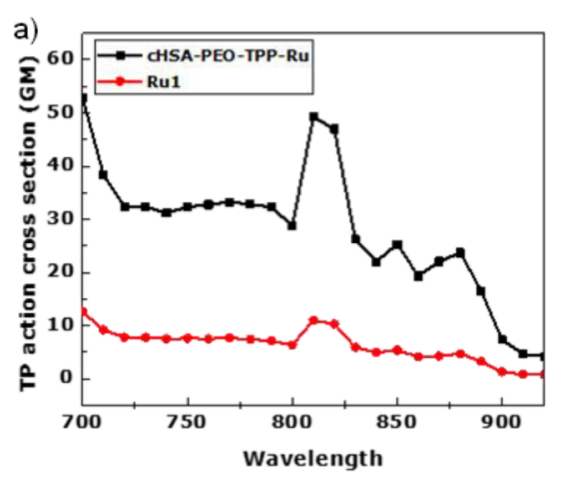

b)

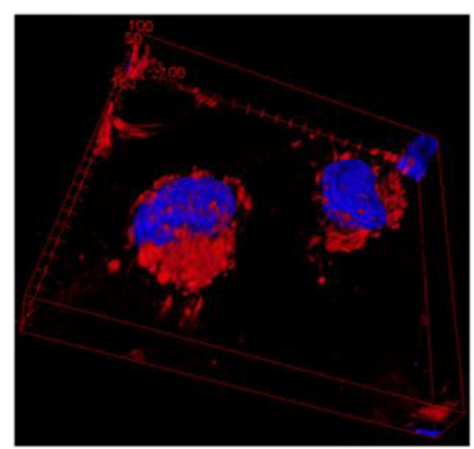

Figure 5. (a) Two-photon absorption cross section of cHSA-PEOTPP-Ru and Rul measured in water at excitation wavelengths from 700 to $920 \mathrm{~nm}$ using Rhodamin B as reference. (b) Two-photon 3D surface projection $z$-stack confocal microscopy image of HeLa cells incubated with cHSA-PEO-TPP-Ru (red, excited at $810 \mathrm{~nm}$ ) and nuclear staining dye Hoechst (blue, excited at $405 \mathrm{~nm}$ ).

Improved TP features are of great relevance to trigger localized photochemical reactions beneath the skin with minimum offtarget photodamage. The 5-times increased value clearly indicates that the design concept of combining multiple $\mathrm{Ru}$ complexes within one nanosized protein transporter allows achieving enhanced TP directed PDT applications. According to previous studies, the TPA cross section of 50 GM obtained for CHSA-PEO-TPP-Ru should be sufficient for its application as 2PA PDT drug candidate. ${ }^{40,41}$ Recent Ru-complexes providing high TP cross sections are based on sophisticated ligand designs or highly charged ligand substituents ${ }^{42,43}$ that influence cell uptake mechanisms and sub cellular distribution patterns as shown by Barton and Puckett. ${ }^{44}$ In case of cHSAPEO-TPP-Ru, a clear three-dimensional (3D) distribution in the cytosol (red) was demonstrated in Figure 5b, where the location of the nucleus was stained with Hoechst dye (3D video, Video SI-V).

\section{CONCLUSION}

We have converted the plasma protein HSA into a highly phototoxic, biodegradable macromolecular PS by controlling its solubility, subcellular targeting pathways, and toxicity. The mitochondria targeted macromolecular PS reported herein revealed significantly enhanced photophysical and chemical properties as well as greatly improved ${ }^{1} \mathrm{O}_{2}$ quantum yields. To the best of our knowledge, the observed phototoxicity was the highest (indicated by the lowest $\mathrm{IC}_{50}$ value) ever reported for Ru-complexes. We believe that high Ru-loading capacity, enhanced cellular uptake efficiency, and localization in mitochondria combined with high photostability and ${ }^{1} \mathrm{O}_{2}$ generation ability contributed to the greatly enhanced cytotoxicity of cHSA-PEO-TPP-Ru. Moreover, effectively blocked cell proliferation and clonogenic potential of the myeloid leukemic cell line OCI-AML3 further underlines the strong antileukemic activity. Intriguingly, preliminary experiments demonstrated less toxicity to normal BM cells, possibly indicating that this biopolymer preferentially targets leukemic cells. These results open the attractive opportunity of treating AML with cHSA-PEO-TPP-Ru-like conjugates using a photodynamic purging step in autologous treatment concepts such as autologous hematopoietic stem cell grafts. ${ }^{45,46}$ The observed TP features of the cHSA-PEO-TPP-Ru biopolymer further provide important prospects for PDT in vivo. The presented strategy to merge a multifunctional protein scaffold and diverse synthetic entities into a versatile nanotransporter platform with tailor-made and potentially synergistic molecular properties could be of great relevance for the preparation of more efficient diagnostic and therapeutic tools in biomedicine.

\section{ASSOCIATED CONTENT}

Supporting Information

The Supporting Information is available free of charge on the ACS Publications website at DOI: 10.1021/jacs.6b13399.

Experimental details and methods; MALDI-ToF spectra; DLS values; absorbance and luminescence decay plots; cell viability studies; ${ }^{1} \mathrm{O}_{2}$ production yields; $\mathrm{CFC}$ and proliferation assays; NMR data (PDF)

Two-photon 3D surface projection $z$-stack of HeLa cells incubated with cHSA-PEO-TPP-Ru (AVI)

\section{AUTHOR INFORMATION}

\section{Corresponding Authors}

*weil@mpip-mainz.mpg.de

*sven.rau@uni-ulm.de

ORCID ${ }^{\circ}$

Tanja Weil: 0000-0002-5906-7205

Notes

The authors declare no competing financial interest.

\section{ACKNOWLEDGMENTS}

The authors are grateful to the financial support of the German Research Foundation (DFG) under P3246029, the ERC Synergy Grant 319130-BioQ, the Horizon2020 project "Hyperdiamond”, and LGFG scholarship from Ulm University.

\section{REFERENCES}

(1) Ogilby, P. R. Chem. Soc. Rev. 2010, 39, 3181-3209.

(2) DeRosa, M. C.; Crutchley, R. J. Coord. Chem. Rev. 2002, 234, 351-371.

(3) Mari, C.; Pierroz, V.; Ferrari, S.; Gasser, G. Chem. Sci. 2015, 6, 2660-2686.

(4) Hamblin, M. R.; Jori, G. Photodynamic Inactivation of Microbial Pathogens; Hamblin, M. R., Jori, G., Eds.; Comprehensive Series in Photochemical \& Photobiological Sciences; The Royal Society of Chemistry: London, 2011.

(5) Wilkinson, F.; Helman, W. P.; Ross, A. B. J. Phys. Chem. Ref. Data 1993, 22, 113-262.

(6) Lovell, J. F.; Liu, T. W. B.; Chen, J.; Zheng, G. Chem. Rev. 2010, 110, 2839-2857. 
(7) Cheng, L.; Wang, C.; Feng, L.; Yang, K.; Liu, Z. Chem. Rev. 2014, 114, 10869-10939.

(8) Sun, B.; Guan, J. X.; Xu, L.; Yu, B.-L.; Jiang, L.; Kou, J. F.; Wang, L.; Ding, X. D.; Chao, H.; Ji, L. N. Inorg. Chem. 2009, 48, 4637-4639.

(9) Marin, V.; Holder, E.; Hoogenboom, R.; Schubert, U. S. Chem. Soc. Rev. 2007, 36, 618-635.

(10) Liu, J.; Chen, Y.; Li, G.; Zhang, P.; Jin, C.; Zeng, L.; Ji, L.; Chao, H. Biomaterials 2015, 56, 140-153.

(11) Arenas, Y.; Monro, S.; Shi, G.; Mandel, A.; McFarland, S.; Lilge, L. Photodiagn. Photodyn. Ther. 2013, 10, 615-625.

(12) Wang, T.; Zabarska, N.; Wu, Y.; Lamla, M.; Fischer, S.; Monczak, K.; Ng, D. Y. W.; Rau, S.; Weil, T. Chem. Commun. 2015, 51, 12552-12555.

(13) Wongrakpanich, A.; Geary, S. M.; Joiner, M. L.; Anderson, M. E.; Salem, A. K. Nanomedicine (London, U. K.) 2014, 9, 2531-2543.

(14) Decaudin, D.; Marzo, I.; Brenner, C.; Kroemer, G. Int. J. Oncol. 1998, 12, 141-152.

(15) Downward, J. Nat. Rev. Cancer 2003, 3, 11-22.

(16) Wang, B.; Galliford, C. V.; Low, P. S. Nanomedicine 2014, 9, 313-330.

(17) Dayem, A. A.; Choi, H.-Y.; Kim, J.-H.; Cho, S.-G. Cancers 2010, 2, 859-884.

(18) Ng, D. Y. W.; Wu, Y.; Kuan, S. L.; Weil, T. Acc. Chem. Res. 2014, 47, 3471-3480.

(19) Wu, Y.; Pramanik, G.; Eisele, K.; Weil, T. Biomacromolecules 2012, 13, 1890-1898.

(20) Wu, Y.; Chakrabortty, S.; Gropeanu, R. a; Wilhelmi, J.; Xu, Y.; Er, K. S.; Kuan, S. L.; Koynov, K.; Chan, Y.; Weil, T. J. Am. Chem. Soc. 2010, 132, 5012-5014.

(21) Wu, Y.; Eisele, K.; Doroshenko, M.; Algara-Siller, G.; Kaiser, U.; Koynov, K.; Weil, T. Small 2012, 8, 3465-3475.

(22) Wu, Y.; Ihme, S.; Feuring-Buske, M.; Kuan, S. L.; Eisele, K.; Lamla, M.; Wang, Y.; Buske, C.; Weil, T. Adv. Healthcare Mater. 2013, 2, 884-894.

(23) Eisele, K.; Gropeanu, R. A.; Zehendner, C. M.; Rouhanipour, A.; Ramanathan, A.; Mihov, G.; Koynov, K.; Kuhlmann, C. R. W.; Vasudevan, S. G.; Luhmann, H. J. Biomaterials 2010, 31, 8789-8801.

(24) Palesch, D.; Boldt, F.; Müller, J. A.; Eisele, K.; Stürzel, C. M.; Wu, Y.; Münch, J.; Weil, T. ChemBioChem 2016, 17, 1504-1508.

(25) Joshi, N. S.; Whitaker, L. R.; Francis, M. B. J. Am. Chem. Soc. 2004, 126, 15942-15943.

(26) Maeda, H.; Wu, J.; Sawa, T.; Matsumura, Y.; Hori, K. J. Controlled Release 2000, 65, 271-284.

(27) Jabir, N. R.; Tabrez, S.; Ashraf, G. M.; Shakil, S.; Damanhouri, G. A.; Kamal, M. A. Int. J. Nanomed. 2012, 7, 4391-4408.

(28) Petermann, L.; Staehle, R.; Pfeifer, M.; Reichardt, C.; Sorsche, D.; Wächtler, M.; Popp, J.; Dietzek, B.; Rau, S. Chem. - Eur. J. 2016, 22, $8240-8253$.

(29) Fong, J.; Kasimova, K.; Arenas, Y.; Kaspler, P.; Lazic, S.; Mandel, A.; Lilge, L. Photochem. Photobiol. Sci. 2015, 14, 2014-2023.

(30) Neugebauer, U.; Pellegrin, Y.; Devocelle, M.; Forster, R. J.; Signac, W.; Moran, N.; Keyes, T. E. Chem. Commun. 2008, 2, 53075309.

(31) Lu, D.; Gai, F.; Qiao, Z.-A.; Wang, X.; Wang, T.; Liu, Y.; Huo, Q. RSC Adv. 2016, 6, 51591-51597.

(32) Stephenson, M.; Reichardt, C.; Pinto, M.; Wächtler, M.; Sainuddin, T.; Shi, G.; Yin, H.; Monro, S.; Sampson, E.; Dietzek, B.; Mcfarland, S. A. J. Phys. Chem. A 2014, 118, 10507-10521.

(33) Reichardt, C.; Pinto, M.; Wächtler, M.; Stephenson, M.; Kupfer, S.; Sainuddin, T.; Guthmuller, J.; McFarland, S. A.; Dietzek, B. J. Phys. Chem. A 2015, 119, 3986-3994.

(34) Breskey, J. D.; Lacey, S. E.; Vesper, B. J.; Paradise, W. A.; Radosevich, J. A.; Colvard, M. D. Photomed. Laser Surg. 2013, 31, 398407.

(35) Liu, D. Bull. Environ. Contam. Toxicol. 1981, 26, 145-149.

(36) Fey, M. F.; Buske, C. Ann. Oncol. 2013, 24, 1-6.

(37) Quentmeier, H.; Martelli, M. P.; Dirks, W. G.; Bolli, N.; Liso, a; Macleod, R. a F.; Nicoletti, I.; Mannucci, R.; Pucciarini, a; Bigerna, B.;
Martelli, M. F.; Mecucci, C.; Drexler, H. G.; Falini, B. Leukemia 2005 19, 1760-1767.

(38) Vegi, N. M.; Klappacher, J.; Oswald, F.; Mulaw, M. A.; Mandoli, A.; Thiel, V. N.; Bamezai, S.; Feder, K.; Martens, J. H. A.; Rawat, V. P. S.; Mandal, T.; Quintanilla-Martinez, L.; Spiekermann, K.; Hiddemann, W.; Döhner, K.; Döhner, H.; Stunnenberg, H. G.; Feuring-Buske, M.; Buske, C. Cell Rep. 2016, 16, 498-507.

(39) Cunderlíková, B. C.; Vasovič, V.; Sieber, F.; Furre, T.; Nesland, J.; Peng, Q. Bone Marrow Transplant. 2010, 45, 1553-1561.

(40) Boca, S. C.; Four, M.; Bonne, A.; van der Sanden, B.; Astilean, S.; Baldeck, P. L.; Lemercier, G. Chem. Commun. 2009, 4590-4592.

(41) Boreham, E. M.; Jones, L.; Swinburne, A. N.; Blanchard-Desce, M.; Hugues, V.; Terryn, C.; Miomandre, F.; Lemercier, G.; Natrajan, L. S. Dalton Trans. 2015, 44, 16127-16135.

(42) Huang, H.; Yu, B.; Zhang, P.; Huang, J.; Chen, Y.; Gasser, G.; Ji, L.; Chao, H. Angew. Chem., Int. Ed. 2015, 54, 14049-14052.

(43) Mongin, O.; Four, M.; Chevreux, S.; Blanchard-Desce, M.; Lemercier, G. Chimia 2015, 69, 666-669.

(44) Puckett, C. A.; Barton, J. K. J. Am. Chem. Soc. 2007, 129, 46-47.

(45) Traul, D. L.; Sieber, F. J. Photochem. Photobiol., B 2015, 153, 153-163.

(46) Lum, L. G.; Yamagami, M.; Giddings, B. R.; Joshi, I.; Schober, S. L.; Sensenbrenner, L. L.; Sieber, F. Blood 1991, 77, 2701-2706. 